Pacific Journal of Mathematic 


\section{MONOTONICITY OF PERMANENTS OF CERTAIN DOUBLY STOCHASTIC MATRICES}

\section{DAVID LONDON}

Let $p_{k}(A), k=1, \cdots, n$, denote the sum of the permanents of all $k \times k$ submatrices of the $n \times n$ matrix $A$.

We prove that

$$
\text { (*) } p_{k}\left(I_{n}+P_{n}\right)=\frac{n}{n-k}\left(\begin{array}{c}
2 n-k-1 \\
k
\end{array}\right), \quad k=1, \cdots, n-1 \text {, }
$$

where $I_{n}$ and $P_{n}$ are respectively the $n \times n$ identity matrix and the $n \times n$ permutation matrix with 1 's in positions $(1,2),(2,3), \cdots,(n-1, n),(n, 1)$. Using $(*)$, we prove that for $n \geqq 3$ and $A=\left(I_{n}+P_{n}\right) / 2$, the functions

$$
p_{k}\left((1-\theta) J_{n}+\theta A\right), \quad k=2, \cdots, n,
$$

are strictly monotonic increasing in the interval $0 \leqq \theta \leqq 1$. Here $J_{n}$ is the $n \times n$ matrix all whose entries are equal to $1 / n$.

Let $A$ be an $n \times n$ matrix, let $p(A)$ be the permanent of $A$, let $p_{k}(A), k=1, \cdots, n$, be the sum of the permanents of all $\left(\begin{array}{l}n \\ k\end{array}\right)^{2} k \times k$ submatrices of $A$ and define $p_{0}(A)=1$. Note that $p_{n}(A)=p(A)$.

Denote by $\Omega_{n}$ the set of all $n \times n$ doubly stochastic matrices, by $J_{n}$ the $n \times n$ matrix all whose entries are equal to $1 / n$, by $I_{n}$ the $n \times n$ identity matrix and by $P_{n}$ the $n \times n$ permutation matrix with 1 's in positions $(1,2),(2,3), \cdots,(n-1, n),(n, 1)$.

The van der Waerden conjecture asserts that if $A \in \Omega_{n}$, then

$$
p(A) \geqq p\left(J_{n}\right)=\frac{n !}{n^{n}},
$$

with equality if and only if $A=J_{n}$.

A stronger version of this conjecture states that the function

$$
p\left((1-\theta) J_{n}+\theta A\right),
$$

where $A$ is any fixed matrix on the boundary of $\Omega_{n}$, is strictly increasing in the interval $0 \leqq \theta \leqq 1$. In [2] the above assertion was proved for $A=I_{n}$ and for $A=\left(n J_{n}-I_{n}\right) /(n-1)$. In [5, p. 158, Problem 8] the problem of finding other matrices $A$, for which the above assertion holds, was posed.

In the present paper we prove this assertion for $A=\left(I_{n}+P_{n}\right) / 2$. We actually prove a stronger result: for $n \geqq 3$ and $A=\left(I_{n}+P_{n}\right) / 2$ the functions 


$$
h_{A, k}(\theta)=p_{k}\left((1-\theta) J_{n}+\theta A\right), \quad k=2, \cdots, n,
$$

are strictly increasing in the interval $0 \leqq \theta \leqq 1$.

We start with the following lemma.

LeMmA 1. Let $n \geqq 3$ and let $A \in \Omega_{n}$. If

$$
\frac{p_{i}(A)}{p_{i}\left(J_{n}\right)} \leqq \frac{p_{i+1}(A)}{p_{i+1}\left(J_{n}\right)}, \quad i=1, \cdots, n-1,
$$

with strict inequality for $1 \leqq i<n-1$, then the functions

$$
h_{A, k}(\theta)=p_{k}\left((1-\theta) J_{n}+\theta A\right), \quad k=2, \cdots, n,
$$

are strictly increasing in the interval $0 \leqq \theta \leqq 1$.

Proof. By [4, Lemma 2],

$$
h_{A, k}(\theta)=p_{k}\left(J_{n}\right) \sum_{i=0}^{k}\left(\begin{array}{l}
k \\
i
\end{array}\right)(1-\theta)^{k-i} \theta^{i} \frac{p_{i}(A)}{p_{i}\left(J_{n}\right)} .
$$

Differentiating, we obtain

$$
h_{A, k}^{\prime}(\theta)=k p_{k}\left(J_{n}\right) \sum_{i=1}^{k-1}\left(\begin{array}{c}
k-1 \\
i
\end{array}\right)(1-\theta)^{k-i-1} \theta^{i}\left(\frac{p_{i+1}(A)}{p_{i+1}\left(J_{n}\right)}-\frac{p_{i}(A)}{p_{i}\left(J_{n}\right)}\right) .
$$

From (1) and (2) follows that

$$
h_{A, k}^{\prime}(\theta)>0, k=2, \cdots, n,
$$

in $0<\theta<1$, and so the functions $h_{A, k}(\theta)$ are strictly increasing in the interval $0 \leqq \theta \leqq 1$.

Dokovic [1] (see also [3]) conjectured that (1) holds for all $A \in$ $\Omega_{n}$. Lemma 1 shows that if the Dokovic conjecture holds for a certain matrix $A \in \Omega_{n}$, then the functions $h_{A, k}(\theta), k=2, \cdots, n$, are increasing in the interval $0 \leqq \theta \leqq 1$.

To apply Lemma 1 for a given $A, p_{k}(A), k=2, \cdots, n$, have to be evaluated. Although the evaluation of $p_{k}(A)$ is in general rather difficult, explicit formulas for $p_{k}(A)$ are obvious for $A=I_{n}$ and can be developed for $A=\left(I_{n}+P_{n}\right) / 2$.

For $A=I_{n}$, we get

$$
p_{k}\left(I_{n}\right)=\left(\begin{array}{l}
n \\
k
\end{array}\right), k=0, \cdots, n .
$$

Noting that

$$
p_{k}\left(J_{n}\right)=\left(\begin{array}{l}
n \\
k
\end{array}\right)^{2} \frac{k !}{n^{k}}, \quad k=0, \cdots, n
$$


(1) follows with strict inequality for $1 \leqq i \leqq n-1$. Hence, for $n \geqq 2$, $p_{k}\left((1-\theta) J_{n}+\theta I_{n}\right), k=2, \cdots, n$, are strictly increasing in $0 \leqq \theta \leqq 1$. For $k=n$, we get the result of Friedland and Minc [2].

To find formulas for $p_{k}\left(I_{n}+P_{n}\right)$, it is convenient first to bring some combinatorial results.

LEMma 2. Let $l$ and $m$ be positive integers, $m \leqq l$. The number $l$ can be represented as a sum of $m$ positive integers in $\left(\begin{array}{r}l-1 \\ m-1\end{array}\right)$ different ways. (Two representations differing in the order of the summands are regarded different.)

Proof. The lemma can be proved easily by induction. We prefer to use power series technique.

Consider

$$
\frac{x}{1-x}=\sum_{r=1}^{\infty} x^{r}, \quad|x|<1 .
$$

It is obvious that the requested number of representations is equal to the coefficient of $x^{l}$ in the power series of $[x /(1-x)]^{m}$, which is easily found to be equal to $\left(\begin{array}{c}l-1 \\ m-1\end{array}\right)$.

Lemma 3. Let $k, l$ and $n$ be positive integers, $k<n$. Then

$$
\begin{gathered}
\sum_{m=1}^{\min (l, n-k)}\left(\begin{array}{c}
l \\
m
\end{array}\right)\left(\begin{array}{c}
n-k-1 \\
n-k-m
\end{array}\right)=\left(\begin{array}{c}
n-k+l-1 \\
n-k
\end{array}\right), \\
\sum_{m=0}^{k}\left(\begin{array}{c}
n-m-1 \\
n-k-1
\end{array}\right)\left(\begin{array}{c}
n-k+m-1 \\
n-k-1
\end{array}\right)=\left(\begin{array}{c}
2 n-k-1 \\
k
\end{array}\right) .
\end{gathered}
$$

Proof. We use again power series.

To prove (4), we consider

$$
\begin{aligned}
(1+x)^{l} & =\sum_{r=0}^{l}\left(\begin{array}{l}
l \\
r
\end{array}\right) x^{r}, \\
(1+x)^{n-k-1} & =\sum_{r=0}^{l}\left(\begin{array}{c}
n-k-1 \\
r
\end{array}\right) x^{r} .
\end{aligned}
$$

The sum in the lefthand side of (4) is equal to the coefficient of $x^{n-k}$ in the power series of $(1+x)^{n-k+l-1}$, which is $\left(\begin{array}{c}n-k+l-1 \\ n-k\end{array}\right)$.

To prove (5), we consider

$$
\frac{x^{n-k-1}}{(1-x)^{n-k}}=\sum_{r=n-k-1}^{\infty}\left(\begin{array}{c}
r \\
n-k-1
\end{array}\right) x^{r}, \quad|x|<1 .
$$


The sum in the lefthand side of (5) is equal to the coefficient of $x^{2 n-k-2}$ in the power series of $\left[x^{n-k-1} /(1-x)^{n-k}\right]^{2}$, which is $\left(\begin{array}{c}2 n-k-1 \\ k\end{array}\right)$. The proof of the lemma is completed.

Let $n$ and $l$ be positive integers, $l \leqq n$. Let $\left(n_{1}, \cdots, n_{l}\right), 1 \leqq$ $n_{1}<n_{2}<\cdots<n_{l} \leqq n$, be a $l$-combination of $1, \cdots, n$. Let $m$ be the number of $r$ 's, $r=1, \cdots, l$, for which $n_{r+1} \neq n_{r}+1$, where $n_{l+1}$ is taken as $n_{1}$ and $n+1$ as 1 . We say that the l-combination $\left(n_{1}, \cdots, n_{l}\right)$ has $m$ gaps. Obviously, $m \leqq l$ and $m+l \leqq n$; i.e., $0 \leqq$ $m \leqq \min (l, n-l)$.

Take $l<n$ and arrange $1, \cdots, n$ in increasing order (clockwise) in a circle. Then the set $\left(n_{1}, \cdots, n_{l}\right)$ and its complement have the same number of (connected) components. This number is the number $m$ defined above as the number of gaps of $\left(n_{1}, \cdots, n_{l}\right)$.

For example, if $n=6$ and $l=3$, then the number of gaps of $(1,2,3)$ and $(1,2,6)$ is 1 , of $(1,3,4)$ is 2 and of $(1,3,5)$ is 3 . If $n=l$, then $m=0$.

We denote by $\left(\begin{array}{c}n \\ l, m\end{array}\right)$ the number of $l$-combinations of $1, \cdots, n$ having $m$ gaps. $\left(\begin{array}{c}n \\ l, m\end{array}\right)$ is thus defined for all nonnegative integers $l, m, n$ satisfying $0<l \leqq n, 0 \leqq m \leqq \min (l, n-l)$. We also define $\left(\begin{array}{c}n \\ 0,0\end{array}\right)=1$. From the definition of $\left(\begin{array}{c}n \\ l, m\end{array}\right)$ follows that

$$
\sum_{m=0}^{\min (l, n-l)}\left(\begin{array}{c}
n \\
l, m
\end{array}\right)=\left(\begin{array}{l}
n \\
l
\end{array}\right)
$$

In the following lemma we obtain a formula for $\left(\begin{array}{c}n \\ l, m\end{array}\right)$.

Lemma 4. Let $l, m, n$ be positive integers satisfying $0<l \leqq$ $n-1,0<m \leqq \min (l, n-l)$. Then

$$
\left(\begin{array}{c}
n \\
l, m
\end{array}\right)=\frac{n}{m}\left(\begin{array}{c}
l-1 \\
m-1
\end{array}\right)\left(\begin{array}{c}
n-l-1 \\
m-1
\end{array}\right)
$$

Proof. $\left(\begin{array}{c}n \\ l, m\end{array}\right)$ is equal to the number of $l$-combinations of $1, \cdots$, $n$ with $m$ gaps. We first find the number of $l$-combinations of the form $\left(1, n_{2}, \cdots, n_{l}\right)$ with $m$ gaps.

Arrange the numbers $1, \cdots, n$ in a circle and take a $l$-combination $\left(1, n_{2}, \cdots, n_{l}\right)$ with $m$ gaps. As $l<n$, the set $\left(1, n_{2}, \cdots, n_{i}\right)$ and its complement have each $m$ components. Let $m_{i}$ and $m_{i}^{\prime}, i=1, \cdots, m$, be the number of elements in the $i$ th component of $\left(1, n_{2}, \cdots, n_{l}\right)$ and its complement respectively. We have 


$$
\left\{\begin{array}{l}
\sum_{i=1}^{m} m_{i}=l \\
\sum_{i=1}^{m} m_{i}^{\prime}=n-l
\end{array}\right.
$$

It is obvious that there is a $1-1$ correspondence between the $l$-combinations of the form $\left(1, n_{2}, \cdots, n_{l}\right)$ with $m$ gaps and the $2 m$ tuples $\left(m_{1}, m_{1}^{\prime}, \cdots, m_{m}, m_{m}^{\prime}\right)$ of positive integers satisfying (7). By Lemma 2 , the number of these $2 m$-triples is $\left(\begin{array}{c}l-1 \\ m-1\end{array}\right)\left(\begin{array}{c}n-l-1 \\ m-1\end{array}\right)$. Hence, the number of $l$-combinations of the form $\left(1, n_{2}, \cdots, n_{l}\right)$ with $m$ gaps is $\left(\begin{array}{c}l-1 \\ m-1\end{array}\right)\left(\begin{array}{c}n-l-1 \\ m-1\end{array}\right)$.

For each of the numbers $1, \cdots, n$ we get $\left(\begin{array}{c}l-1 \\ m-1\end{array}\right)\left(\begin{array}{c}n-l-1 \\ m-1\end{array}\right)$ $l$-combinations with $m$ gaps. Assembling all these combinations, each combination with $l$ gaps is repeated $m$ times. Hence, to get the number of these combinations, $\left(\begin{array}{c}n-1 \\ m-1\end{array}\right)\left(\begin{array}{c}n-l-1 \\ m-1\end{array}\right)$ has to multiplied by $n$ and divided by $m$. Formula (6) is thus proved.

In the following lemma we obtain formulas for $p_{k}\left(I_{n}+P_{n}\right), k=$ $0, \cdots, n$.

Lemma 5. Let $n \geqq 2$. Then

$$
p_{k}\left(I_{n}+P_{n}\right)= \begin{cases}\frac{n}{n-k}\left(\begin{array}{c}
2 n-k-1 \\
k
\end{array}\right), & k=0, \cdots, n-1, \\
2 & k=n .\end{cases}
$$

Proof. Formula (8) is easily verified for $k=0$ and $k=n$.

Let $1 \leqq k \leqq n-1 . \quad p_{k}\left(I_{n}+P_{n}\right)$ is equal to the number of different diagonals of 1's of length $k$ in $I_{n}+P_{n}$. (Where diagonals of length $k$ in the $n \times n$ matrix $I_{n}+P_{n}$ are defined in the obvious way.) Each such diagonal is composed of $l$ elements of $I_{n}$ and $k-l$ elements of $P_{n}$.

Let $\left(n_{1}, \cdots, n_{l}\right)$ be a l-combination of $1, \cdots, n$ with $m$ gaps. The number of 1's in $P_{n}$ belonging either to the rows $n_{1}, \cdots, n_{l}$ or to the columns $n_{1}, \cdots, n_{l}$ is $l+m$. Hence, the diagonal of length $l$ consisting of 1 's in positions $\left(n_{1}, n_{1}\right),\left(n_{2}, n_{2}\right), \cdots,\left(n_{l}, n_{l}\right)$ can be augmented, using elements of $P_{n}$, to $\left(\begin{array}{c}n-l-m \\ k-l\end{array}\right)$ different diagonals of 1 's of length $k$. As there are $\left(\begin{array}{c}n \\ l, m\end{array}\right) l$-combinations with $m$ gaps, the number of diagonal of length $k$ which originate in a $l$-combination with $m$ gaps is $\left(\begin{array}{c}n \\ l, m\end{array}\right)\left(\begin{array}{c}n-l-m \\ k-l\end{array}\right)$. Summing up over all possible $m$ and $l$, we obtain 


$$
p_{k}(A)=\sum_{l=0}^{k} \sum_{m=0}^{\min (l, n-k)}\left(\begin{array}{c}
n \\
l, m
\end{array}\right)\left(\begin{array}{c}
n-l-m \\
k-l
\end{array}\right) .
$$

Noting that $\left(\begin{array}{c}n \\ 0,0\end{array}\right)=1$ and, as $k<n,\left(\begin{array}{c}n \\ l, 0\end{array}\right)=0$ for $l=1, \cdots, k$, it follows that

$$
p_{k}(A)=\left(\begin{array}{l}
n \\
k
\end{array}\right)+\sum_{l=1}^{k} \sum_{m=1}^{\min (l, n-k)}\left(\begin{array}{c}
n \\
l, m
\end{array}\right)\left(\begin{array}{c}
n-l-m \\
k-l
\end{array}\right) .
$$

Using now Lemma 4, we obtain

$$
p_{k}(A)=\left(\begin{array}{l}
n \\
k
\end{array}\right)+\sum_{l=1}^{k} \sum_{m=1}^{\min (l, n-k)} \frac{n}{m}\left(\begin{array}{c}
l-1 \\
m-1
\end{array}\right)\left(\begin{array}{c}
n-l-1 \\
m-1
\end{array}\right)\left(\begin{array}{c}
n-l-m \\
k-l
\end{array}\right) .
$$

As

$$
\begin{aligned}
& \frac{n}{m}\left(\begin{array}{c}
l-1 \\
m-1
\end{array}\right)\left(\begin{array}{c}
n-l-1 \\
m-1
\end{array}\right)\left(\begin{array}{c}
n-l-m \\
k-l
\end{array}\right) \\
& \quad=\frac{n}{l}\left(\begin{array}{l}
n-l-1 \\
n-k-1
\end{array}\right)\left(\begin{array}{c}
l \\
m
\end{array}\right)\left(\begin{array}{l}
n-k-1 \\
n-k-m
\end{array}\right),
\end{aligned}
$$

it follows that

$$
p_{k}(A)=\left(\begin{array}{l}
n \\
k
\end{array}\right)+n \sum_{l=1}^{k} \frac{1}{l}\left(\begin{array}{c}
n-l-1 \\
n-k-1
\end{array}\right) \sum_{m=1}^{\min (l, n-k)}\left(\begin{array}{c}
l \\
m
\end{array}\right)\left(\begin{array}{l}
n-k-1 \\
n-k-m
\end{array}\right),
$$

and using (4), we obtain

$$
p_{k}(A)=\left(\begin{array}{l}
n \\
k
\end{array}\right)+n \sum_{l=1}^{k} \frac{1}{l}\left(\begin{array}{c}
n-l-1 \\
n-k-1
\end{array}\right)\left(\begin{array}{c}
n-k+l-1 \\
n-k
\end{array}\right) .
$$

But

$$
\frac{1}{l}\left(\begin{array}{c}
n-k+l-1 \\
n-k
\end{array}\right)=\frac{1}{n-k}\left(\begin{array}{c}
n-k+l-1 \\
n-k-1
\end{array}\right) .
$$

So

$$
\begin{aligned}
p_{k}(A) & =\left(\begin{array}{l}
n \\
k
\end{array}\right)+\frac{n}{n-k} \sum_{l=1}^{k}\left(\begin{array}{c}
n-l-1 \\
n-k-1
\end{array}\right)\left(\begin{array}{c}
n-k+l-1 \\
n-k-1
\end{array}\right) \\
& =\frac{n}{n-k} \sum_{l=0}^{k}\left(\begin{array}{l}
n-l-1 \\
n-k-1
\end{array}\right)\left(\begin{array}{c}
n-k+l-1 \\
n-k-1
\end{array}\right) .
\end{aligned}
$$

Formula (8) follows from (5) and (9).

We bring now our main result.

THEOREM. Let $n \geqq 3$ and let $A=\left(I_{n}+P_{n}\right) / 2$. Then the functions 


$$
h_{A, k}(\theta)=p_{k}\left((1-\theta) J_{n}+\theta A\right), \quad k=2, \cdots, n,
$$

are strictly increasing in the interval $0 \leqq \theta \leqq 1$.

Proof. By Lemma 1, it is sufficient to show that

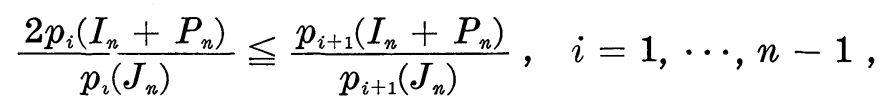

with strict inequality for $1 \leqq i<n-1$.

For $i=n-1$, (10) holds with equality sign.

For $i=1, \cdots, n-2,(3)$ and (8) imply

$$
\frac{p_{i+1}\left(I_{n}+P_{n}\right)}{p_{i+1}\left(J_{n}\right)}=\frac{(i+1) ![(n-i-1) !]^{2} n^{i+2}}{(n-i-1)(n !)^{2}}\left(\begin{array}{c}
2 n-i-2 \\
i+1
\end{array}\right) .
$$

From (11) follows

$$
\begin{aligned}
& \frac{p_{i+1}\left(I_{n}+P_{n}\right)}{p_{i+1}\left(J_{n}\right)}-\frac{2 p_{i}}{\left.i_{n}+P_{n}\right)} \\
& \quad=\frac{2 i n^{i+1}(2 n-i-2) ![(n-i-1) !]^{2}(n-i-1)}{(n !)^{2}(2 n-2 i-1) !} .
\end{aligned}
$$

Hence (10) holds with strict inequality for $1 \leqq i<n-1$, and the proof of our theorem is completed.

We note that the theorem holds also for all $n \times n$ matrices $A$ which can be obtained from $\left(I_{n}+P_{n}\right) / 2$ by permutations of rows and columns.

Acknowledgment. I wish to thank Professor C. J. Smyth for his useful remarks.

\section{REFERENCES}

1. D. Z. Doković, On a conjecture by van der Waerden, Mat. Vesnik (19) 4 (1967), 272-276.

2. S. Friedland and H. Minc, Monotonicity of permanents of doubly stochastic matrices, Linear and Multilinear Algebra, 6 (1978), 227-231.

3. D. London, On the Dokovic conjecture for matrices of rank two, to appear in Linear and Multinear Algebra.

4. M. Marcus and H. Minc, Extensions of classical matrix inequalities, Linear Algebra and Appl., 1 (1968), 421-444.

5. H. Minc, Permanents, Encyclopedia of Mathematics and its Applications, Vol. 6, Addison-Wesley, 1978.

Received January 9, 1980.

University of British Columbia

Vancouver, B.C. V6T 1Y4

TECHNION, I.I.T.

Haifa, IsRael (Current address) 



\section{PACIFIC JOURNAL OF MATHEMATICS}

\section{EDITORS}

DONALD BABBITT (Managing Editor)

University of Galifornia

Los Angeles, California 90024

Hugo RossI

University of Utah

Salt Lake City, UT 84112

C. C. MOORE AND ANDREW OGG

University of California

Berkeley, CA 94720
J. DugundJI

Department of Mathematics University of Southern California Los Angeles, California 90007

R. FinN and J. Milgram Stanford University Stanford, California 94305

\section{ASSOCIATE EDITORS}

R. ARENS

E. F. BeCKenbaCh

B. H. NEUManN

F. WOLF

K. YosHIDA

\section{SUPPORTING INSTITUTIONS}

UNIVERSITY OF ARIZONA

UNIVERSITY OF BRITISH COLUMBIA

CALIFORNIA INSTITUTE OF TECHNOLOGY

UNIVERSITY OF CALIFORNIA

MONTANA STATE UNIVERSITY

UNIVERSITY OF NEVADA, RENO

NEW MEXICO STATE UNIVERSITY

OREGON STATE UNIVERSITY
UNIVERSITY OF OREGON

UNIVERSITY OF SOUTHERN CALIFONIA

STANFORD UNIVERSITY

UNIVERSITY OF HAWAII

UNIVERSITY OF TOKYO

UNIVERSITY OF UTAH

WASHINGTON STATE UNIVERSITY

UNIVERSITY OF WASHINGTON 


\section{Pacific Journal of Mathematics}

\section{Vol. 95, No. $1 \quad$ September, 1981}

John Allen Beachy and William David Blair, On rings with bounded

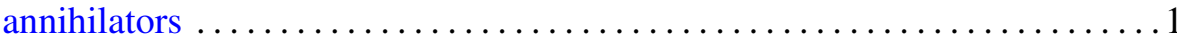

Douglas S. Bridges, A constructive look at positive linear functionals on

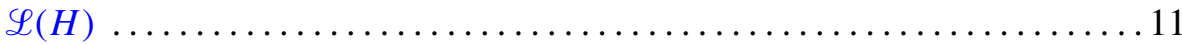

Muneo Chō and Makoto Takaguchi, Boundary points of joint numerical ranges

W. J. Cramer and William O. Ray, Solvability of nonlinear operator equations

Lester Eli Dubins and Gideon Schwarz, Equidiscontinuity of

Borsuk-Ulam functions

Maria Fragoulopoulou, Spaces of representations and enveloping 1.m.c.

*-algebras

Robert F. Geitz and J. Jerry Uhl, Jr., Vector-valued functions as families of scalar-valued functions

Ross Geoghegan, The homomorphism on fundamental group induced by a homotopy idempotent having essential fixed points

Ross Geoghegan, Splitting homotopy idempotents which have essential fixed points

Paul Jacob Koosis, Entire functions of exponential type as multipliers for

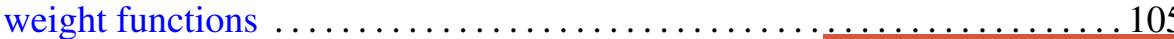

David London, Monotonicity of permanents of certain doubly stochastic matrices

Howard J. Marcum, Two results on cofibers

Giancarlo Mauceri, Zonal multipliers on the Heisenberg group

Edward Wilfred Odell, Jr. and Y. Sternfeld, A fixed point theorem in $c_{0} \quad \ldots 161$

Bernt Karsten Oksendal, Brownian motion and sets of harmonic measure zero

Andrew Douglas Pollington, The Hausdorff dimension of a set of normal numbers

Joe Repka, Base change lifting and Galois invariance ...

Gerald Suchan, Concerning the minimum of permanents on doubly stochastic circulants

Jun-ichi Tanaka, On isometries of Hardy spaces on compact abelian groups

Aaron R. Todd, Quasiregular, pseudocomplete, and Baire spaces 